\title{
Three-Dimensional Quantification of the Effects between Different Types of RME
}

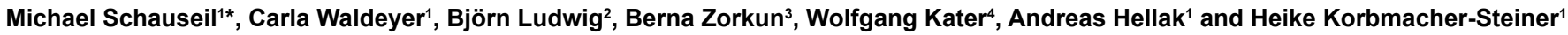

${ }^{1}$ University of Marburg, Germany, Georg-Voigt-Str. 3, 35039 Marburg, Germany

${ }^{2}$ Orthodontic praxis, Germany, 56841 Traben-Trarbach and University of Saarland, Germany

${ }^{3}$ Trakya University, 22030 Edirne, Turkey

${ }^{4}$ Maxillofacial surgical practice, Hessenring 128, 61348 Bad Homburg vor der Höhe, Germany

\begin{abstract}
Objectives: To analyse if Hybrid-RME has different effects on skeletal, dental and periodontal aspects when compared with those of Hyrax-RME or Hyrax-SARME.

Methods: In this retrospective multicentre-study 28 patients with a severe nasomaxillary constriction were treated either with a Hyrax-type RME (6 patients, $13.92 \pm 0.36$ years), with a Hybrid-RME (7 patients, $17.20 \pm 2.80$ years) or with a surgical assisted Hyrax-SARME (15 patients, $30.58 \pm 9.26)$. This study was performed in analogy to the method published by Garib, et al. using the freeware OsiriX 4.0. All measurements were performed twice with an interval of two weeks and the calculated mean value of both measurements was used for statistical evaluation, which was performed using SPSS 21 for Mac.

Results: The age differences between all groups were significant $(p<0.001)$. All appliances led to a significant skeletal and dental expansion while the buccal bone lamella reduced in dependence of the employed appliance. Effects on the suture were comparable at the canine region while they were significantly differences at the molar region, where Hyrax-RME scored first, Hybrid-RME scored second and Hyrax-SARME had the least effects. While Hyrax-RME led to the greatest, Hyrax-SARME showed the least dental expansion at premolar and molar level. Hyrax- and Hybrid-RME showed significantly enhanced skeletal effects on the maxillary base and the skeletal nose than Hyrax-SARME. HyraxSARME had significantly less (more than half that much) molar tilting as Hybrid-RME $\left(0.84^{\circ} \mathrm{vs} .1 .74^{\circ} / \mathrm{mm}\right.$ activation). Hybrid-RME tended to show the least bone loss (4-12\%) while Hyrax-SARME tended to show the most (20-30\%).

Conclusions: In patients with a borderline-age (17-20 years) Hybrid-RME might be a promising alternative for Hyrax-RME or Hyrax-SARME to gain skeletal and dental expansion while maintaining the surrounding periodontal bones. Future studies are necessary to investigate if also a surgically assisted Hybrid-SARME might be beneficial in older patients $(20+)$ to combine the parallel dental expansion with the pronounced skeletal effects while maintaining the surrounding bones.
\end{abstract}

Keywords: Ossification; Hybrid-RME; Hyrax-type RME; HyraxSARME

\section{Introduction}

Recent studies stated that after a certain point of ossification, conventional dental-borne RME doesn't fulfil the requirements of maximal skeletal and dental effects while sparing the surrounding periodontal tissues at the same time [1]. At this point, supportive surgical weakening (SARME) still provides positive orthodontic and orthopaedic effects and should therefore be applied especially for outgrown adults [1-3]. However there are always patients with borderline-age (in between adolescence and adult maturation) where the practitioner can't be sure if conventional RME might be adequate or a surgical weakening is indicated [4]

The implementation of skeletal anchorage into RME treatment started using the "Dresden-Distractor" [5,6], which reduced the negative side effects associated with tooth-borne RME [6]. The main disadvantage of this appliance was, that beside a mini-screw at one side of the palate, the Dresden-Distractor needed a Straumann-implant (with a diameter of $3.5 \mathrm{~mm}$ ), which made bone-predrilling sequences necessary $[5,6]$. Furthermore earlier publications focused on effects in combination with previous surgical weakening [5-11].

Hybrid-RME (also called Hybrid-Hyrax) was implemented to overcome the disadvantage of requiring a $3.5 \mathrm{~mm}$ Straumann implant [12]. It just needed two mini-screws placed at the anterior palate [13], whereby the procedure could be performed easily in local anaesthesia [4,14-17]. Previous studies proved that Hybrid-RME has particular beneficial impact on class III treatment [14,15,17] and furthermore suggested that it might also be a successful alternative to conventional appliances because of its expanding forces at the centre of resistance of the nasomaxillary complex [16-18]. For the practitioner it is of particular interest at what age a specific RME-modification should be preferred, whereby the aim would always be a maximum of therapeutic effectiveness while being minimal invasive.

A study compared skeletal and dental effects of Hyrax-RME and Hybrid-RME in young adults with the same age (13-14 years) [19]. The only significant difference was that the bone thicknesses of the left first premolars significantly differed between groups. Although HybridRME therefore seemed to have advantages over Hyrax-RME, it should be critically questioned if the insertion of mini-implants is justified, considering the fact, that Hyrax-RME was able to lead to a comparable skeletal and dental expansion at that age.

*Corresponding author: Michael Schauseil, Orthodontist, Philipps-Universitat Marburg, Germany, Georg-Voigt-Str. 3, 35039 Marburg, Germany, Tel: 06421-5866716; E-mail: schausei@med.uni-marburg.de

Received July 29, 2015; Accepted August 18, 2015; Published August 28, 2015

Citation: Schauseil M, Waldeyer C, Ludwig B, Zorkun B, Kater W, et al. (2015) Three-Dimensional Quantification of the Effects between Different Types of RME. Dentistry 5: 328. doi:10.4172/2161-1122.1000328

Copyright: (c) 2015 Schauseil M, et al. This is an open-access article distributed under the terms of the Creative Commons Attribution License, which permits unrestricted use, distribution, and reproduction in any medium, provided the original author and source are credited. 
Because in late adolescents dental RME has its limitations in skeletal expansion [20] and Hyrax-SARME is certainly invasive, it is of particular interest to investigate if Hybrid-RME might still have comparable positive effects at borderline-age.

Therefore, the following questions should be answered in this study:

a. Do skeletal, dental and periodontal effects significantly differ between the three approaches of transverse widening?

b. Which RME modification achieves the most wanted therapeutic effect?

c. Which RME modification caused the worst side effects?

d. Does Hybrid-RME or surgical weakening reduce possible side effects?

\section{Materials and Methods}

This is a retrospective multi-centre study comprised of 28 patients. All patients had a severe nasomaxillary constriction and were treated either with a Hyrax-type RME (6 patients) or with a Hybrid-RME (7 patients) or with surgical assisted Hyrax-SARME (15 patients) with same appliance design as Figure 1. To achieve maximal therapeutic effects while being minimal invasive, all groups had different average ages. Patients with Hyrax-RME were the youngest (13.92 \pm 0.36 years), followed by patients with Hybrid-RME (17.20 \pm 2.80 years). Patients who underwent surgical weakening were the oldest (30.58 \pm 9.26 years).

One of the investigators (Berna Zorkun, Cumhuriyet University, Sivas, Turkey) performed conventional therapy and one (Wolfgang Kater, Private Practice, Bad Homburg, Germany) performed surgical therapy. 10 patients were men, 18 patients were women. This majority of female gender was present in all subgroups.

Each patient was instructed to activate the Hyrax-screw 3 times a day, so that a daily expansion of $0.60 \mathrm{~mm}$ was carried out. The activation amount was carried out according to the individual needs. The RMEappliance remained in situ as a retention device for a minimum of three to six months without further activation. In all patients threedimensional documentation was conducted (low dose CT or CBCT before (T0) vs. after maximal activation (T1)).

This retrospective analysis of the 3D-datasets was reviewed and approved by the ethics commission of the Saarland (Homburg, Germany, Approval number 170/12 from 29.11.2012).

The inclusion criteria of the study are

- No syndromes

- Severe Maxillary transverse deficiency with need for RME or SARME

- Proper 3D-dataset at T0 and T1 (Low-dose CT or CBCT, no artefacts, adequate Field-of-view)

- Successful RME-procedure (mediate Diastema after 5 days)

- Treatment either with Hyrax-RME, Hybrid-RME or HyraxSARME

The exclusion criteria include

- Patients with syndromes

- No sufficient 3D-datasets available

- Different radiograph methods between T0 and T1 (Low-dose$\mathrm{CT} / \mathrm{CBCT})$
This study was performed in analogy to the method published by Garib et al. [21]. All the measurements (points, distances and angles) are explained in Table 1.

As in previous publications for examining the image data the freeware, OsiriX 4.0 (Pixmeo, Bern, Switzerland) was used [21-23]. All measurements were performed twice with an interval of two weeks and the calculated mean value of both measurements was used for statistical evaluation. Statistical analysis was performed using SPSS 21 for Mac. Normal distribution was tested with Shapiro-Wilk test and graphical data output. If normal distribution was given, parametric tests were done. If normal distribution wasn't present, nonparametric tests were performed. Intergroup differences were analysed with Kruskal-Wallis and subsequent Mann-Whitney-U tests. The intraoperator reliability was measured using Spearman Rho and Pearson correlation. For all tests the significance level was set to $\mathrm{p}<0.05$.

\section{Results}

\section{Reproducibility}

The intraoperator correlation was very high for all measurements. Spearman-Rho tests showed a highly significant correlation for the skeletal distances and dental angles (correlation $>0.99, \mathrm{p}<0.001$ ). Pearson-tests revealed highly significant correlation for the dental distances and periodontal bone changes (correlation $>0.98, \mathrm{p}<0.001$ ).

\section{Patient population and RME-activation}

The age differences between all groups were highly significant $(p<0.001)$. The age differences between all groups were significant $(p<0.001)$. Hybrid-RME patients, nonetheless, were significantly older than Hyrax-RME patients $(\mathrm{p}<0.001)$.

Overall, no significant differences concerning RME-activation were present between the studied groups $(\mathrm{p}=0.391)$. Since the activations were slightly different $(6.26+-1.89 \mathrm{~mm})$ and the activation should be ruled out as a potential disruptive factor, all measured values were converted to a change-quotient $\Delta$ as recommended by Motro [13] and Bazargani [24].

Change-
quotient $\Delta=$

This change-quotient $\Delta$ is taking into account the patientdependent individual activation of the expansion screw and therefore describing the treatment effects which result due to $1 \mathrm{~mm}$ appliance activation.

\section{Therapeutic effects}

All appliances led to a significant skeletal and dental expansion (Table 2) while the employed appliance differently affected the buccal

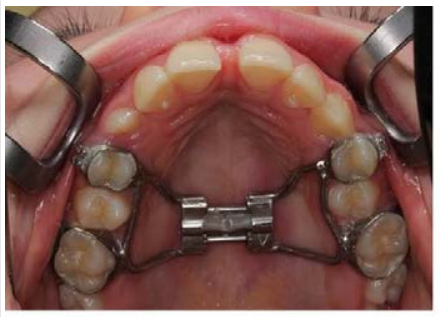

Hyrax-type RME

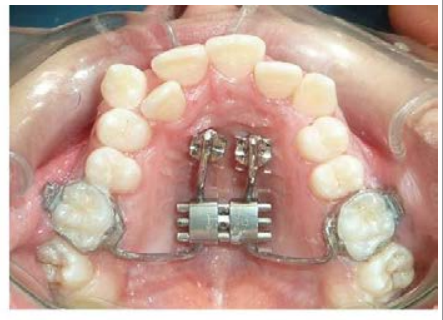

Hybrid-RME
Figure 1: 13 patients received conventional treatment with a Hyrax-RME. 15 additional patients received previous surgical weakening. 7 patients received conventional treatment with Hybrid-RME. 


\begin{tabular}{|c|c|c|}
\hline & Type & Definition \\
\hline \multicolumn{3}{|l|}{ Skeletal } \\
\hline Baseline & Distance & It is defined by the lowest points on both inferior glenoids of the nasal meatus. \\
\hline Maxillary Base & Distance & It is defined from the transverse extension of the baseline to the right and left maxillary alveolar process. \\
\hline Suture opening canines & Distance & It describes the width of the midpalatal suture in CT-sectional area at the level of the canines. \\
\hline Suture opening molars & Distance & It describes the width of the midpalatal suture in CT-sectional area at the level of the upper first molars. \\
\hline Skeletal nose & Distance & $\begin{array}{l}\text { It defines the maximum transverse width of the nasal skeleton at the level of the lower nasal passages. It is parallel to } \\
\text { the baseline and is limited by the inside of the bony lateral nasal wall on both sides. }\end{array}$ \\
\hline \multicolumn{3}{|l|}{ Dental } \\
\hline Apical distance Premolars & Distance & It describes the distance through the apex centers of the palatal roots of 14 and 24 . \\
\hline Coronal distance Premolars & Distance & It describes the distance through the palatal cusps of 14 and 24 . \\
\hline Apical distance Molars & Distance & It describes the distance through the palatal roots of 16 and 26. \\
\hline Coronal distance Molars & Distance & It describes the distance through the mesio-palatal cusps of 16 and 26 . \\
\hline Molar Tipping & Angle & This angle defines the inclination of the maxillary first molar's palatal roots to the reference "baseline". \\
\hline \multicolumn{3}{|l|}{ Periodontal } \\
\hline Bucco-apical bone change 14 & Distance & It describes the distance between the center of the buccal root apex of 14 and the alveolar process. \\
\hline Bucco-apical bone change 24 & Distance & It describes the distance between the center of the buccal root apex of 24 and the alveolar process. \\
\hline Bucco-apical bone change 16 & Distance & It describes the distance between the center of the mesiobuccal root apex of 16 and the alveolar process. \\
\hline Bucco-apical bone change 26 & Distance & It describes the distance between the center of the mesiobuccal root apex of 26 and the alveolar process. \\
\hline
\end{tabular}

Table 1: All measurements were performed in analogy to the study of Garib, et al. [19] and subdivided into skeletal, dental and periodontal effects.

\begin{tabular}{|c|c|c|c|c|c|c|c|}
\hline \multirow{2}{*}{$\begin{array}{c}\text { Relative change } \Delta \text { (effects per } \mathrm{mm} \\
\text { RME-activation) }\end{array}$} & \multicolumn{2}{|c|}{ Hyrax-RME } & \multicolumn{2}{|c|}{ Hyrax-SARME } & \multicolumn{2}{|c|}{ Hybrid-RME } & \multirow{2}{*}{$\begin{array}{c}\begin{array}{c}\text { Intergroup } \\
\text { differences }\end{array} \\
\text { p-value }\end{array}$} \\
\hline & Mean & Std. Deviation & Mean & Std. Deviation & Mean & Std. Deviation & \\
\hline \multicolumn{8}{|l|}{ Skeletal } \\
\hline Baseline & 0.61 & 0.14 & 0.17 & 0.14 & 0.44 & 0.16 & 0.001 \\
\hline Maxillary Base & 0.48 & 0.18 & 0.12 & 0.35 & 0.34 & 0.15 & 0.009 \\
\hline Suture opening canines & 0.64 & 0.32 & 0.59 & 0.4 & 0.54 & 0.28 & 0.844 \\
\hline Suture opening molars & 1.09 & 0.35 & 0.2 & 0.15 & 0.44 & 0.21 & 0.001 \\
\hline Skeletal nose & 0.48 & 0.17 & 0.09 & 0.19 & 0.47 & 0.18 & 0.001 \\
\hline \multicolumn{8}{|l|}{ Dental } \\
\hline Apical distance Premolars & 0.86 & 0.29 & 0.58 & 0.43 & 0.77 & 0.15 & 0.099 \\
\hline Coronal distance Premolars & 1.34 & 0.18 & 1.01 & 0.51 & 0.79 & 0.16 & 0.006 \\
\hline Apical distance Molars & 0.93 & 0.2 & 0.44 & 0.27 & 0.61 & 0.2 & 0.005 \\
\hline Coronal distance Molars & 1.4 & 0.23 & 0.76 & 0.39 & 1.3 & 0.35 & 0.003 \\
\hline Molar Tipping & 0.98 & 0.79 & 0.82 & 1.04 & 1.74 & 0.31 & 0.053 \\
\hline \multicolumn{8}{|l|}{ Periodontal } \\
\hline Bucco-apical bone change 14 & -0.15 & 0.08 & -0.2 & 0.18 & -0.08 & 0.12 & 0.598 \\
\hline Bucco-apical bone change 24 & -0.17 & 0.09 & -0.21 & 0.15 & -0.04 & 0.08 & 0.014 \\
\hline Bucco-apical bone change 16 & -0.22 & 0.33 & -0.27 & 0.22 & -0.05 & 0.06 & 0.032 \\
\hline Bucco-apical bone change 26 & -0.18 & 0.1 & -0.3 & 0.2 & -0.12 & 0.05 & 0.033 \\
\hline
\end{tabular}

Table 2: Descriptive statistics and Kruskal-Wallis-Test summarizing the relative changes $\Delta$.

bone lamella. There were significantly differences in the effects between all three appliances.

\section{Skeletal Effects}

RME-treatment in all three study groups led to a significant widening of the baseline. Hyrax-RME and Hybrid-RME showed significantly larger effects on the baseline than SARME treatment $(\mathrm{p}<0.05)$ (Figure 2).

Hyrax-RME achieved the greatest effect on the maxillary base, Hybrid-RME placed second while SARME had the least impact $(\mathrm{p}<0.006)$ (Figure 3). Both Hyrax-RME and Hybrid-RME led to a significantly higher widening of the skeletal nose $(\mathrm{p}=0.001)$. There was no significant difference between both conventional appliances in regard to their effects on the skeletal nose $(\mathrm{p}=0.945)$ (Figure 4).

All appliances led to a comparable widening of the maxillary suture at the canine level. There was no significant difference between them
( $p>0.67$ ) (Figure 5). However significant differences were detected at concerning suture opening at molar level: while Hyrax-RME had the significantly strongest effects $(\mathrm{p}<0.01)$, Hybrid-RME had less powerful effects. Still Hybrid-RME was significantly stronger than HyraxSARME $(\mathrm{p}=0.009)$ (Figure 6).

\section{Dental Effects}

All three appliances led to a comparable apical dental expansion at the premolar area. There was no statistical significant difference ( $p>0.1)$. Hyrax-RME led to a higher dental expansion at the coronal premolar area than both other appliances $(p<0.012)$ (Figure 7$)$. There was no statistical significant difference between Hybrid-RME and Hyrax-SARME $(\mathrm{p}=0.237)$.

Both Hyrax-SARME and Hybrid-RME led to a significant minor transverse apical expansion in the molar region $(\mathrm{p}=0.022)$. There was no statistical significant difference between Hybrid-RME and Hyrax- 


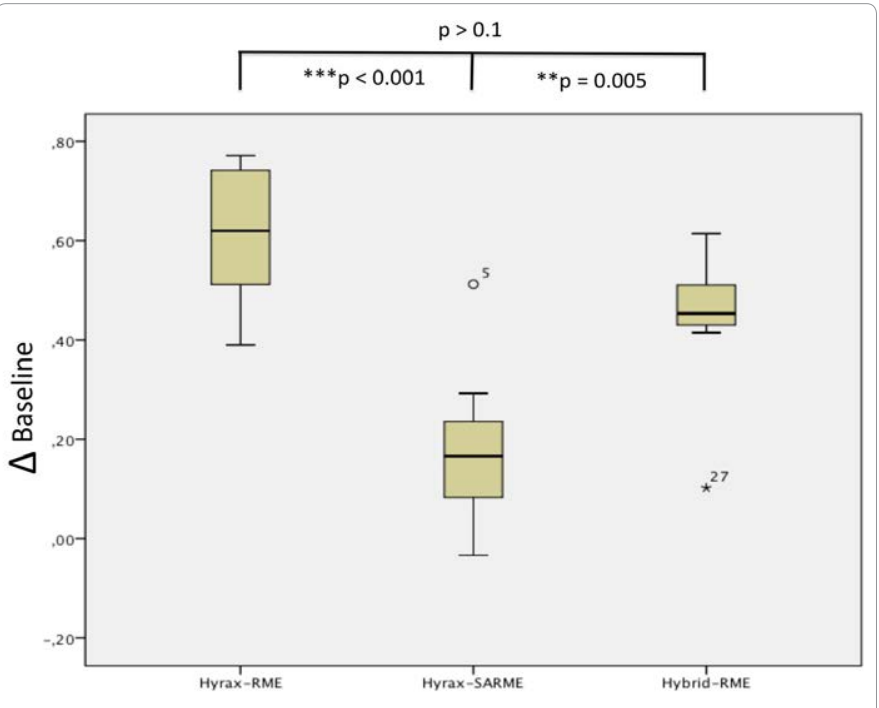

Figure 2: Hyrax-RME and Hybrid-RME showed significantly larger effects on the baseline than Hyrax-SARME treatment.

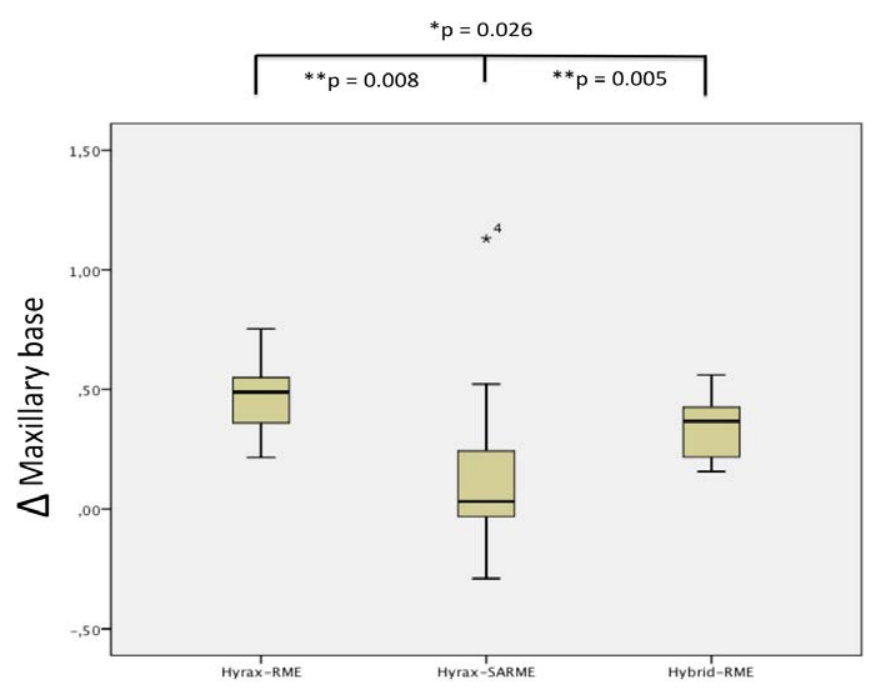

Figure 3: Hyrax-RME achieved the greatest effect on the maxillary base, Hybrid-RME placed second while Hyrax-SARME had the least impact.

SARME. Hyrax-RME and Hybrid-RME led to a significantly higher expansion at the coronal molar level than Hyrax-SARME $(\mathrm{p}<0.005)$. There was no significant difference between Hybrid-RME and HyraxRME. Hybrid-RME showed the highest tipping effects at the first upper molar area (Figure 8). This was significant in comparison to HyraxSARME ( $\mathrm{p}=0.026)$. There was no significant difference between HyraxRME and Hyrax-SARME ( $\mathrm{p}=0.733)$.

\section{Periodontal Effects}

Hybrid-RME tended to spare the buccal bone lamella. While there was no statistical significant difference at 14 ( $\mathrm{p}>0.366)$, it was significant at 24 $(\mathrm{p}<0.022)$ (Figure 9). Furthermore Hybrid-RME showed less buccal bone loss in the apical area of 16 and 26 than both other appliances (Figures 10). This difference was highly significant in comparison to Hyrax-SARME $(p=0.017)$ but not in comparison to Hyrax-RME $(p>0.101)$.

\section{Discussion}

For our study, we used OsiriX software as it was suggested in comparable publications [21-23]. Due to the retrospective design of this study both low-dose CT and CBCT were analysed because both methods proved to be accurate for 3D-measurements [25]. Additionally for precision, only those patients were included with the same type of radiograph method at $\mathrm{T} 0$ and $\mathrm{T} 1$.

All measurements, which were adopted and modified according to Garib et al. [21], showed very high reproducibility which is consistent with the literature [26]. Although there were no significant differences concerning the activations between all three types of RME, we analysed the relative changes as recommended by Motro [13] and Bazargani [24] to enhance the comparability even further. This statistical accuracy was not taken into account in earlier studies $[1,26,27]$ and might therefore be a reason for possible errors of previous publications.

Our results suggest that Hyrax-RME had the strongest expansive effects when applied at premature age. For every $1 \mathrm{~mm}$ activation, it led to a skeletal enlargement between 48 and 109\%, while at the dental level it

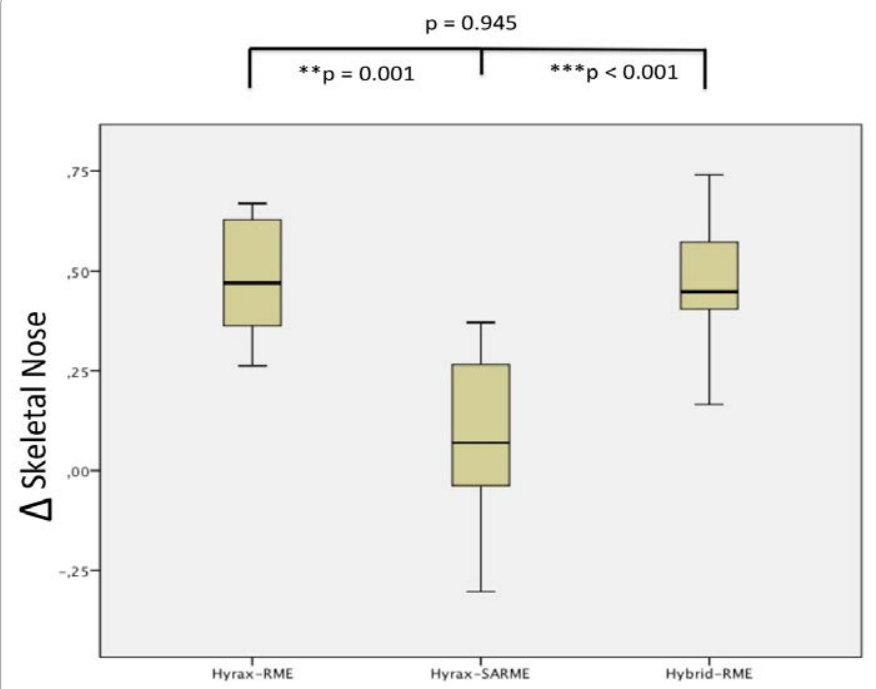

Figure 4: Both Hyrax-RME and Hybrid-RME led to a significantly higher widening of the skeletal nose.

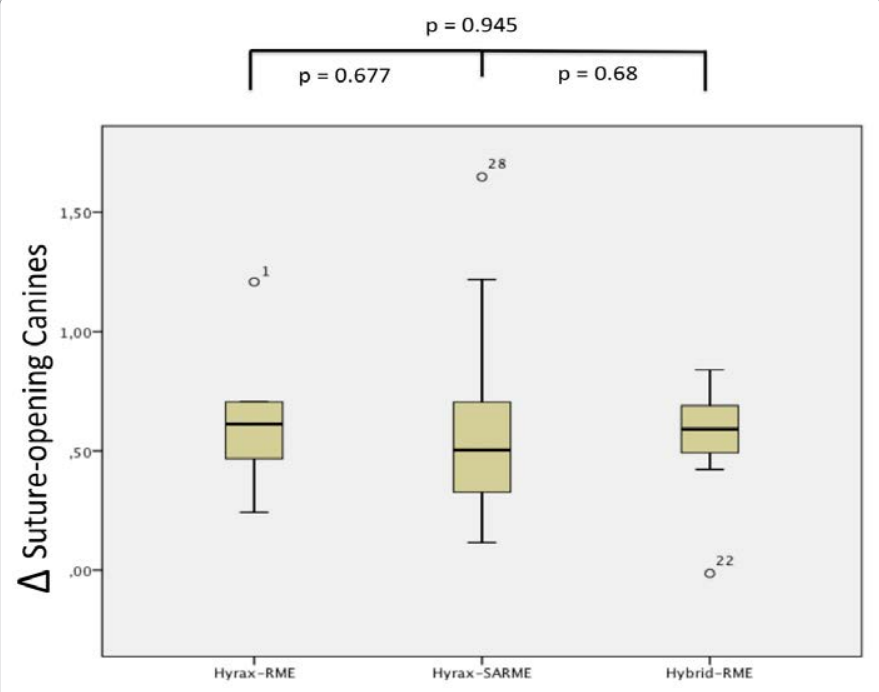

Figure 5: All appliances led to a comparable widening of the maxillary suture at the canine level. 


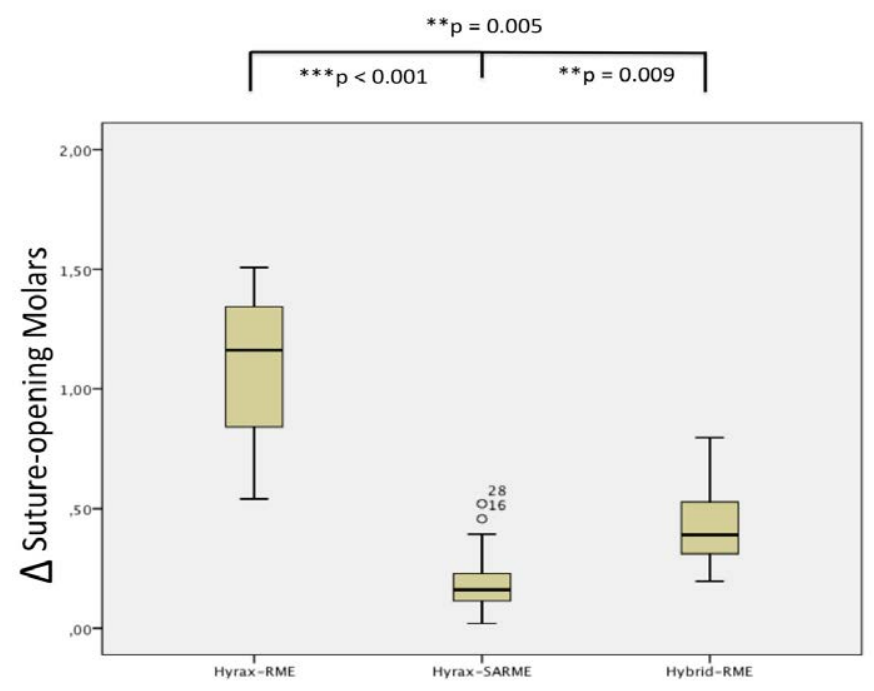

Figure 6: The suture opening at molar level was significantly different, where Hyrax-RME had the strongest and Hybrid-RME less powerful effects. Nevertheless Hybrid-RME was significantly stronger than Hyrax-SARME.
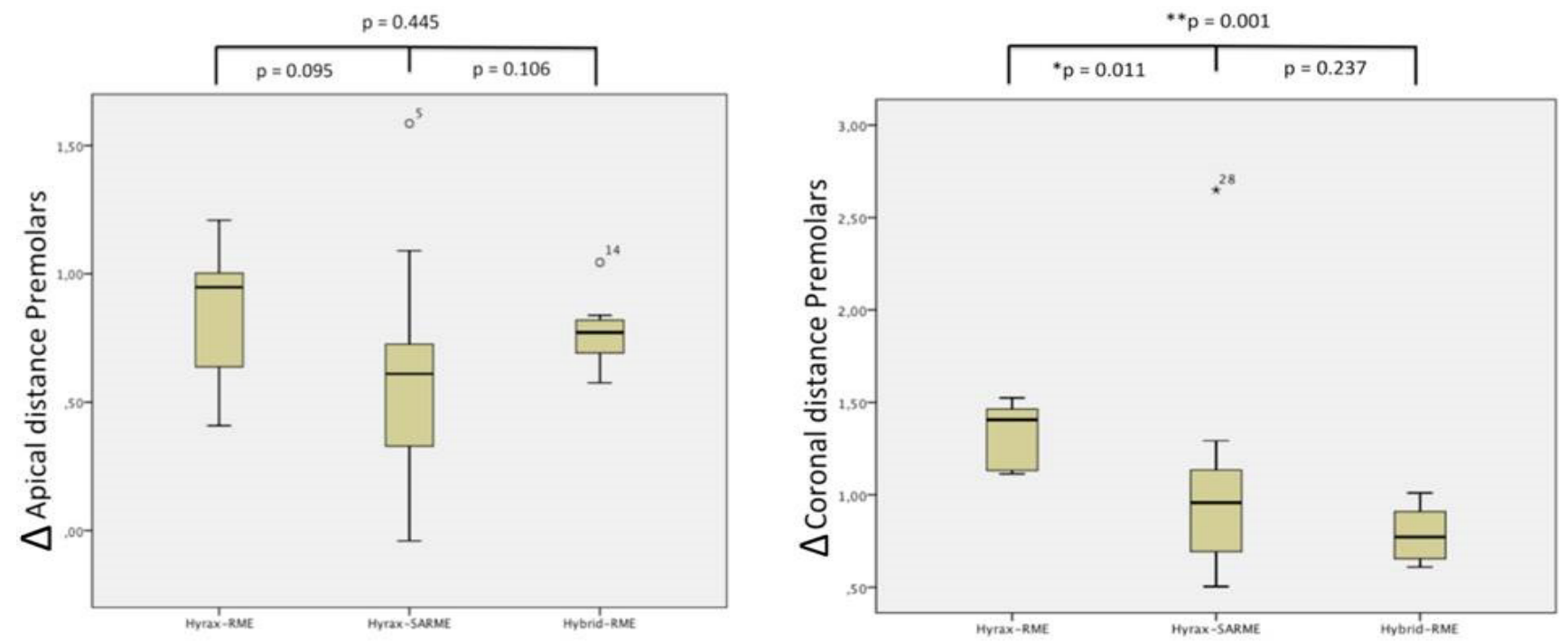

Figure 7: All three appliances led to a comparable apical dental expansion at the premolar area. There was no statistical significant difference. Hyrax-RME led to a higher dental expansion at the coronal premolar area than both other appliances. There was no statistical significant difference between Hybrid-RME and HyraxSARME.

resulted in an expansion between 86 and $140 \%$ with a pronounced level of tilting at the premolar area and an average tilting at the molar level.

In our study Hybrid-RME patients had a borderline-age (17.20 \pm 2.80 years). Nevertheless our treatments led to a significant skeletal $(\Delta=34-54 \%)$ and dental expansion ( $\Delta=61-130 \%)$, whereby HyraxSARME and Hybrid-RME patients showed a V-shaped opening pattern with larger skeletal effects in the canine area $(\Delta=54-59 \%)$ and less skeletal effects in the molar area $(\Delta=20-44 \%)$. In Hyrax-RME patients this situation reversed.

Hyrax-SARME and Hybrid-RME patients showed an opening of the midpalatal suture between $(\Delta=) 20 \%$ and $59 \%$, what stays in accordance with a current review of Bazargani et al., who found a mean sutural expansion between $(\Delta=) 22 \%-53 \%$ with a large variation in the opening pattern [24]. The highly significant $(p<0.006)$ stronger effects of Hyrax-RME at the posterior suture might be explained due to the significant younger age in that group and the hereby decreased ossification level [28].

Furthermore Hybrid-RME showed the least tilting in the premolar and the highest tilting in the molar area. This can be explained with the appliance depended missing of dental anchorage in the premolar area [12] and the direct transmission of force at the centre of resistance of the nasomaxillary complex [16]. Nonetheless Hybrid-RME showed more than twice as powerful skeletal effects $(\Delta=44 \%)$ at the premolar area than Hyrax-SARME $(\Delta=20 \%)$.

It is said that the rate of expansion and retention time doesn't have significant association to buccal bone losses after SARME [26]. In accordance with this we could show that there was no significant correlation between the rate of expansion and bone losses. However 

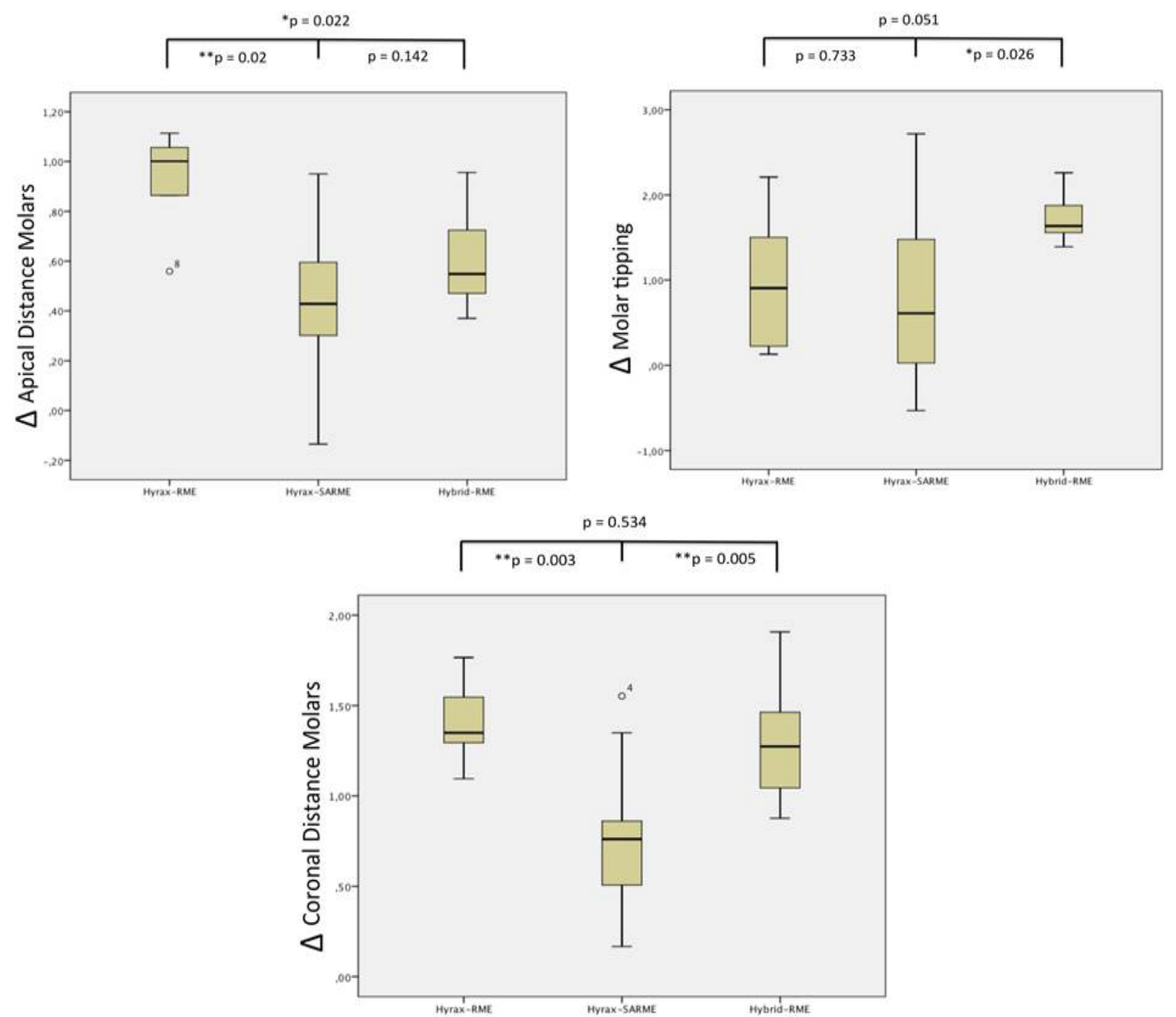

Figure 8: Both Hyrax-SARME and Hybrid-RME led to a significant minor transverse apical expansion in the molar region. Hyrax-RME and Hybrid-RME led to a significantly higher expansion at the coronal molar level than Hyrax-SARME. Hybrid-RME showed the highest tipping effects at the first upper molar area.
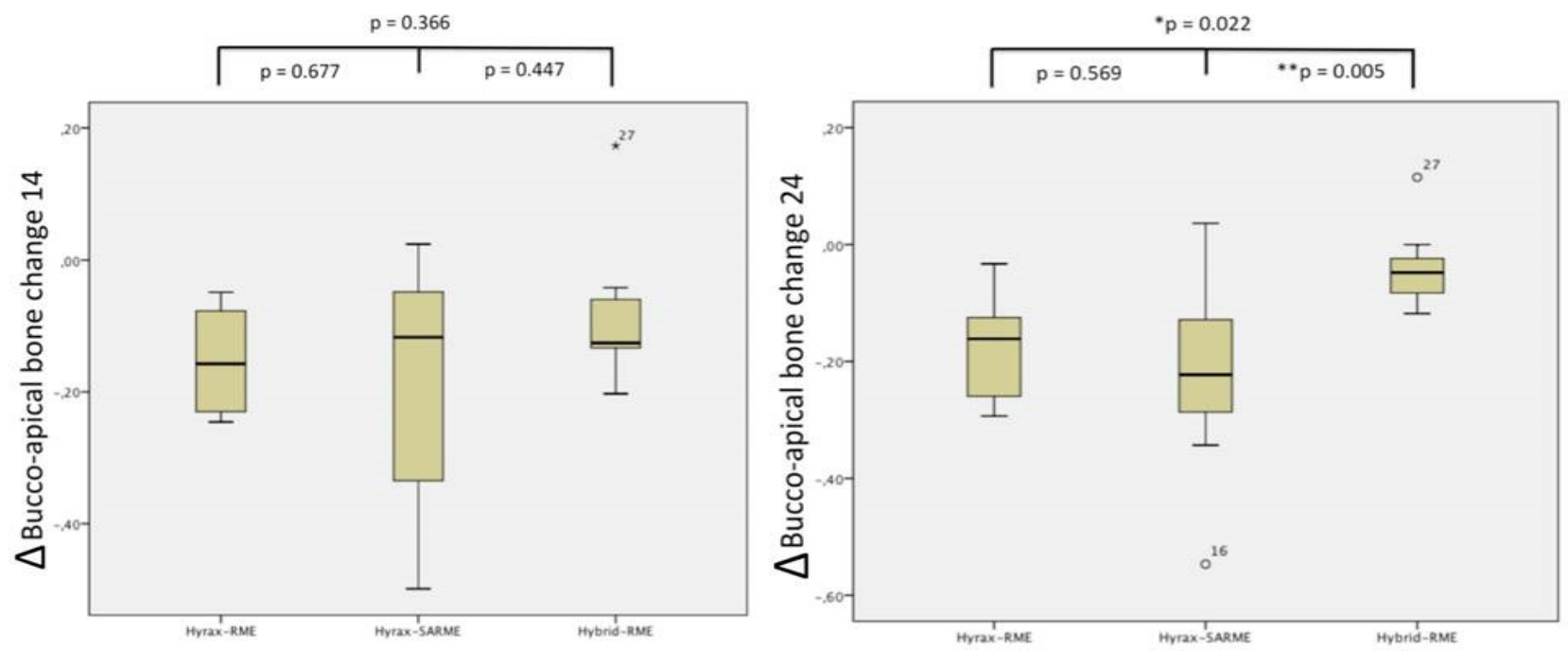

Figure 9: Hybrid-RME tended to spare the buccal bone lamella. While there was no statistical significant difference at 14 it was significant at 24 . 

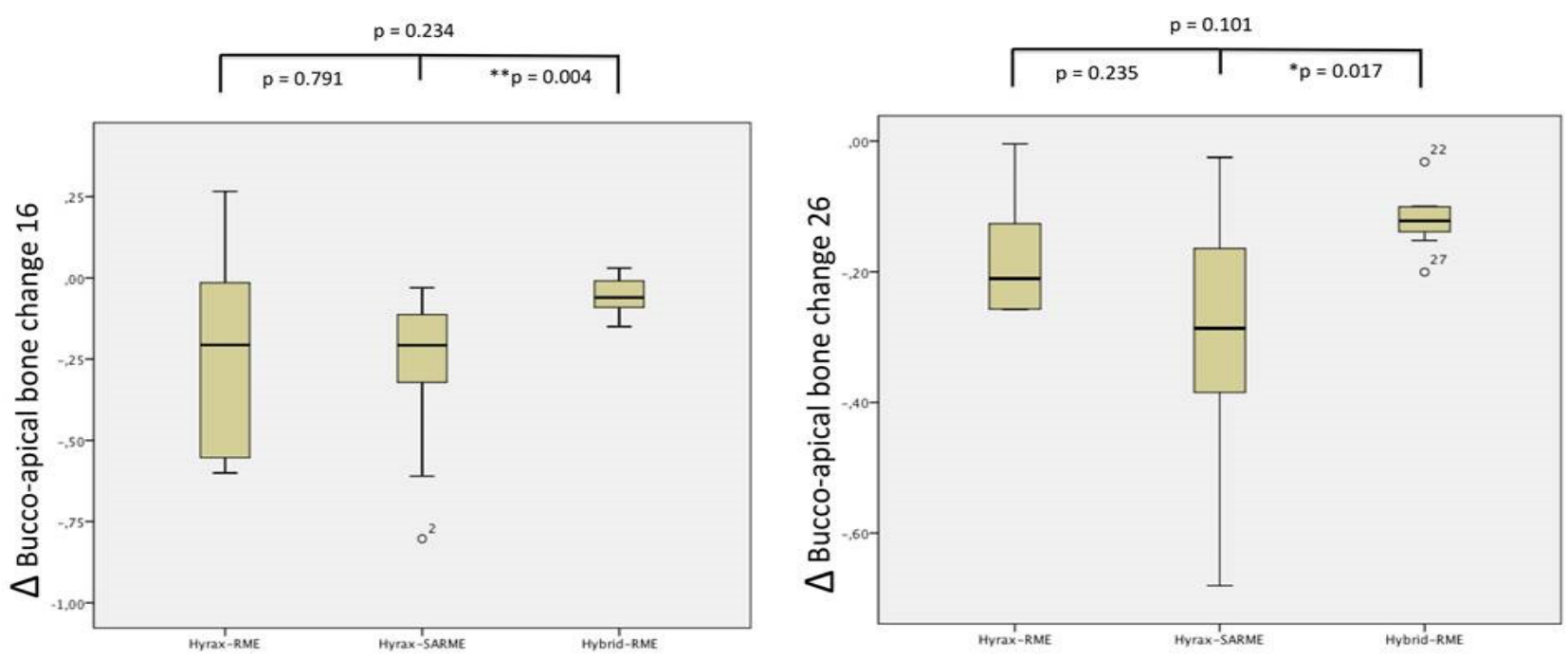

Figure 10: Hybrid-RME showed less buccal bone loss in the apical area of 16 and 26 than both other appliances, whereby this difference was even significant in comparison to Hyrax-SARME.

the clinical conclusion of a recently published study, stating that "SARME does not affect the health status of the periodontal tissues" should subsequently be critically discussed [27].

Our results suggest that, no matter what appliance investigated, buccal bone loss occurred, which was overall between $(\Delta=) 4-30 \%$. However Hybrid-RME tended to prevent these negative side effects both for premolar and molar region with the smallest mean losses (which were between 4 and 12\%) and these trends were even partially significant in comparison to both other appliances. While HyraxRME scored an average result $(\Delta=) 15-22 \%$ in all four analysed areas, Hyrax-SARME showed pronounced buccal bone resorption of $(\Delta=) 20-30 \%$. Bone loss leads to a deterioration of the periodontal situation. In consequence there are plenty of studies in Periodontology and Implantology dealing with approaches to improve bone sparing $[29,30]$. Our results lead to the conclusion that Hybrid-RME was able to achieve a significant skeletal and dental expansion while stressing the surrounding bones the least at the same time, even though it was applied in borderline-age patients.

Laudemann et al. showed that surgically assisted dental borne devices tended to show an increase in transverse widening but at the price of greater attachment loss [31]. Our study leads to the supposition, that also in surgical cases the implementation of mini-screws (HybridSARME) could help to prevent attachment losses, as Hybrid-RME does in conventional cases. Further studies are necessary to prove this hypothesis.

\section{Conclusions}

With the results of our study we can conclude that both Hyraxand Hybrid-RME showed significantly stronger skeletal effects on the maxillary base and the skeletal nose than Hyrax-SARME. Hyrax-SARME had significantly less molar tilting effects as Hybrid-RME ( $\Delta=0.84^{\circ}$ vs. $\Delta=1.74^{\circ}$ ). Hybrid-RME tended to show the least bone losses $(\Delta=4-12 \%)$ while Hyrax-SARME tended to show the most $(\Delta=20-30 \%)$. In young adolescents Hyrax-RME seems to be adequate, in patients with borderlineage (17-20 years) Hybrid-RME might have certain advantages and in older adults (20+) surgical weakening seems to be justified.

\section{Acknowledgment}

The authors are grateful to Ms. Natascha Mainusch (New York, NYC, USA) for editing the English of the study.

\section{References}

1. Sygouros A, Motro M, Ugurlu F, Acar A (2014) Surgically assisted rapid maxillary expansion: cone-beam computed tomography evaluation of different surgical techniques and their effects on the maxillary dentoskeletal complex. Am J Orthod Dentofacial Orthop 146: 748-757.

2. Magnusson A (2013) Evaluation of surgically assisted rapid maxillary expansion and orthodontic treatment. Effects on dental, skeletal and nasal structures and rhinological findings. Swed Dent J Suppl 229: 1-104.

3. Gamage SN, Goss AN (2013) Surgically-assisted rapid maxillary expansion of narrowed maxillae: a case-cohort study. Aust Orthod J 29: 21-27.

4. Schauseil M, Ludwig B, Zorkun B, Hellak A, Korbmacher-Steiner H (2014) Density of the midpalatal suture after RME treatment - a retrospective comparative low-dose CT-study. Head Face Med 10: 18.

5. Tausche E, Hansen L, Hietschold V, Lagravère MO, Harzer W (2007) Threedimensional evaluation of surgically assisted implant bone-borne rapid maxillary expansion: a pilot study. Am J Orthod Dentofacial Orthop 131: S92-S99.

6. Hansen L, Tausche E, Hietschold V, Hotan T, Lagravère M, et al. (2007) Skeletally-anchored rapid maxillary expansion using the Dresden Distractor. J Orofac Orthop 68: 148-158.

7. Tausche E, Hansen L, Schneider M, Harzer W (2008) Bone-supported rapid maxillary expansion with an implant-borne Hyrax screw: the Dresden Distractor. Orthod Fr 79: 127-135.

8. Deeb W, Hansen L, Hotan T, Hietschold V, Harzer W, et al. (2010) Changes in nasal volume after surgically assisted bone-borne rapid maxillary expansion. Am J Orthod Dentofacial Orthop 137: 782-789.

9. Harzer W, Reusser L, Hansen L, Richter R, Nagel T, et al. (2010) Minimally invasive rapid palatal expansion with an implant-supported hyrax screw. Biomed Tech (Berl) 55: 39-45.

10. Petrick S, Hothan T, Hietschold V, Schneider M, Harzer W, et al. (2011) Bone density of the midpalatal suture 7 months after surgically assisted rapid palatal expansion in adults. Am J Orthod Dentofacial Orthop 139: S109-S116.

11. Tausche E, Deeb W, Hansen L, Hietschold V, Harzer W, et al. (2009) CT analysis of nasal volume changes after surgically-assisted rapid maxillary expansion. J Orofac Orthop 70: 306-317.

12. Wilmes B, Nienkemper M, Drescher D (2010) Application and effectiveness of a mini-implant- and tooth-borne rapid palatal expansion device: the hybrid hyrax. World J Orthod 11: 323-330. 
Citation: Schauseil M, Waldeyer C, Ludwig B, Zorkun B, Kater W, et al. (2015) Three-Dimensional Quantification of the Effects between Different Types of RME. Dentistry 5: 328. doi:10.4172/2161-1122.1000328

13. Motro M, Schauseil M, Ludwig B, Zorkun B, Mainusch S, et al. (2015) Rapidmaxillary-expansion induced rhinological effects: a retrospective multicenter study. Eur Arch Otorhinolaryngol.

14. Nienkemper M, Wilmes B, Franchi L, Drescher D, et al. (2014) Effectiveness of maxillary protraction using a hybrid hyrax-facemask combination: A controlled clinical study. Angle Orthod.

15. Nienkemper M, Wilmes B, Pauls A, Drescher D (2013) Maxillary protraction using a hybrid hyrax-facemask combination. Prog Orthod 14: 5.

16. Ludwig B, Baumgaertel S, Zorkun B, Bonitz L, Glasl B, et al. (2013) Application of a new viscoelastic finite element method model and analysis of miniscrew-supported hybrid hyrax treatment. Am J Orthod Dentofacial Orthop 143: 426-435.

17. Wilmes B, Nienkemper M, Ludwig B, Kau CH, Drescher D (2011) Early Class III treatment with a hybrid hyrax-mentoplate combination. J Clin Orthod 45: 15-21.

18. Wilmes B, Ngan P, Liou EJ, Franchi L, Drescher D (2014) Early class II facemask treatment with the hybrid hyrax and Alt-RAMEC protocol. J Clin Orthod 48: 84-93.

19. Gunyuz Toklu M, Germec-Cakan D, Tozlu M (2015) Periodontal, dentoalveolar, and skeletal effects of tooth-borne and tooth-bone-borne expansion appliances. Am J Orthod Dentofacial Orthop 148: 97-109.

20. Lin L, Ahn HW, Kim SJ, Moon SC, Kim SH et al. (2015) Tooth-borne vs bone-borne rapid maxillary expanders in late adolescence. Angle Orthod 85: 253-262.

21. Garib DG, Henriques JF, Janson G, de Freitas MR, Fernandes AY (2006) Periodontal effects of rapid maxillary expansion with tooth-tissue-borne and tooth-borne expanders: a computed tomography evaluation. Am J Orthod Dentofacial Orthop 129: 749-758.

22. Sicurezza E, Palazzo G, Leonardi R (2011) Three-dimensional computerized tomographic orbital volume and aperture width evaluation: a study in patients treated with rapid maxillary expansion. Oral Surg Oral Med Oral Pathol Oral Radiol Endod 111: 503-507.

23. Leonardi R, Sicurezza E, Cutrera A, Barbato E (2011) Early post-treatment changes of circumaxillary sutures in young patients treated with rapid maxillary expansion. Angle Orthod 81: 36-41.

24. Bazargani F, Feldmann I, Bondemark L (2013) Three-dimensional analysis of effects of rapid maxillary expansion on facial sutures and bones. Angle Orthod 83: 1074-1082.

25. Gaia BF, Pinheiro LR, Umetsubo OS, Costa FF, Cavalcanti MG (2014) Validity of three-dimensional computed tomography measurements for Le Fort osteotomy. Int J Oral Maxillofac Surg 43: 197-203.

26. Rungcharassaeng K, Caruso JM, Kan JY, Kim J, Taylor G (2007) Factors affecting buccal bone changes of maxillary posterior teeth after rapid maxillary expansion. Am J Orthod Dentofacial Orthop 132: 428.

27. Jensen T, Johannesen LH, Rodrigo-Domingo M (2015) Periodontal changes after surgically assisted rapid maxillary expansion (SARME). Oral Maxillofac Surg.

28. Melsen B (1975) Palatal growth studied on human autopsy material. A histologic microradiographic study. Am J Orthod 68: 42-54.

29. Graves DT, Li J, Cochran DL (2011) Inflammation and uncoupling as mechanisms of periodontal bone loss. J Dent Res 90: 143-153.

30. Palti A, Hoch T (2002) A concept for the treatment of various dental bone defects. Implant Dent 11: 73-78.

31. Laudemann K, Santo G, Revilla C, Harth M, Kopp S, et al. (2011) Assessment of surgically assisted rapid maxillary expansion regarding pterygomaxillary disjunction using thin volume-rendering technique: in variance analysis and in reliability, accuracy, and validity. J Oral Maxillofac Surg 69: 2631-2643. 